\title{
STRESS-LIFE CURVE FOR HIGH AND LOW CYCLE FATIGUE
}

\author{
Andrzej Kurek, Marta Kurek, Tadeusz tagoda \\ Opole University of Technology, Faculty of Mechanical Engineering, Opole, Poland \\ e-mail: a.kurek@po.opole.pl; ma.kurek@po.opole.pl; t.lagoda@po.opole.pl
}

\begin{abstract}
The paper contains a proposal of a new model for the stress-life curve. The new model reflects the nature of the stress curve occurring under current conditions, which means its shape of an inclined letter $S$. It can be used for both low and high cycle fatigue. Verification of the proposed model was performed on experimental results of ten various materials from three main types that are low-alloy, high-alloy and non-alloy steels.
\end{abstract}

Keywords: stress-life curves, steels and alloys, low-cycle fatigue, high-cycle fatigue

\section{Introduction}

Fatigue is a phenomenon occurring when a material is subject to cyclic loading changing in time and thus burdened with varying stresses. The fatigue phenomenon arises if changes in material properties are caused by these varying loads or stresses, when the material is subject to damage or failure. Fatigue - in terms of the material - is a phenomenon with hazardous inclinations while loads that do not exceed the yield strength can cause a decrease in strength properties or it can even lead to damage of the material. Usually, this happens unexpectedly, under considerably lower stresses than the ultimate tensile strength and, therefore, it can pose a risk to the safety of machine functioning as well as to human life. It is widely known that vast majority of damage is arising during exploitation and its nature is the fatigue. Therefore, it is crucial to calculate the time to failure or values of the maximum stress at which no failure will occur.

The loading history acting on the material can either be random or can indicate certain reproducibility, and then it is termed as cyclic. A particular case of the cyclic load condition is the sinusoidal loading. The fatigue properties of construction materials or components are determined on its basis. Fatigue processes should be also considered in machines subjected to cyclic loading, especially if their proper functioning puts a shadow on human's health or life. Thus, it is especially important in such industrial sectors as aviation (e.g. turbine components), automotive (e.g. engine and suspension components) or power engineering (e.g. boilers, pipelines).

From the standpoint of determining fatigue strength, the understanding of fatigue characteristics is of crucial meaning. For design calculations of materials in terms of their fatigue life, cyclic tests on specimens are performed in laboratories. There are several types of characteristics that can be distinguished. The most common characteristics are stress-based fatigue characteristics (recommended for the high cycle fatigue) and strain-based fatigue characteristics (suggested for the low cycle fatigue). Considering the structure of the new model of fatigue characteristics, which is proposed by the authors, this study focuses explicitly on the stress-based fatigue characteristics. Keeping in mind the fact that in engineering practice the stress rather than the strain approach to design is much more common, this research work attempts to employ the stress-life curve to describe also the low cycle fatigue properties of the material. Moreover, on the basis of experiences gained by other researchers, it is noticed that stress-based fatigue characteristics, best describing the experimental points, should rather be adopted as an inclined letter $S$ than a straight line. The model proposed in this work satisfies this condition. 


\section{Review of stress-based fatigue characteristics}

In the research literature on fatigue life one can find many suggestions related to stress-based fatigue models. The most well-known characteristics are these proposed by Wöhler (1858) or Basquin (1910). However, some authors propose slightly different and more complex models.

Fatigue diagram (S-N curve) developed by Wöhler in 1860 has its longest history. The fatigue model is written as

$$
\log N_{f}=a-b \sigma_{a}
$$

or when the base-logarithm will be replaced with the natural logarithm as

$$
\ln N_{f}=a-b \sigma_{a}
$$

where: $N_{f}$ - fatigue life in cycles (number of cycles to fatigue failure), $\sigma_{a}$ - stress amplitude, $a, b$ - parameters of the equation (constants of the model of regression).

The curve model proposed in 1910 by Basquin is basically a modified S-N curve model by Wöhler. In the research literature, this model is more commonly used and often is known under the name of the author of the pioneer model, and it is expressed by

$$
\log N_{f}=a-b \log \sigma_{a}
$$

Like in the S-N curve model by Wöhler also in the research literature and in calculations performed in real life, the base-logarithm is substituted by the natural logarithm. Basquin introduced to the S-N curve by Wöhler the log of the amplitude of stress $\sigma_{a}$.

Another analysed fatigue characteristics estimated in 1914 was Stromeyer's suggestion (Stromeyer, 1914)

$$
\log N_{f}=a-b \log \left(\sigma_{a}-\sigma_{a f}\right)
$$

where: $\sigma_{a}$-stress (or strain) amplitude, $\sigma_{a f}$ - fatigue limit.

A subsequent fatigue model was developed in 1955 by Corson (Palin-Luc, 1996)

$$
N_{f}=\frac{a}{\sigma_{a}-\sigma_{a f}} \exp \left[-c\left(\sigma_{a}-\sigma_{a f}\right)\right]
$$

or as a result of logarithmisation performed on both sides of the equation

$$
\ln N_{f}=\ln a-\ln \left(\sigma_{a}-\sigma_{a f}\right)-c\left(\sigma_{a}-\sigma_{a f}\right)
$$

The next fatigue model is the dependence proposed in the work by Bastenaire (1972)

$$
N_{f}=\frac{a}{\sigma_{a}-\sigma_{a f}} \exp \left[-\left(\frac{\sigma_{a}-\sigma_{a f}}{b}\right)^{c}\right]
$$

or after logarithmisation performed on both sides of the equation

$$
\ln N_{f}=\ln a-\ln \left(\sigma_{a}-\sigma_{a f}\right)-\left(\frac{\sigma_{a}-\sigma_{a f}}{b}\right)^{c}
$$

Whereas, in 1999 an other approach was proposed by Pascual and Meeker (1999) and expressed by the equation

$$
\ln N_{f}=a+b \log \left(\sigma_{a}-\sigma_{a f}\right)+\varepsilon
$$

where: $\varepsilon$ - error term. 
Substituting $a+\varepsilon$ for a constant, Eq. (2.9) takes the same form as in Stromeyer's model, except that Pascual and Meeker suggest application of the natural logarithm, therefore

$$
\ln N_{f}=a-b \log \left(\sigma_{a}-\sigma_{a f}\right)
$$

In the same year, Kato and Hayashi (1999) introduced the dependence

$$
\log \sigma_{a}=a-b \log \left(\log N_{f}\right)
$$

Whereas, Kohout (2000) introduced a new model taking into account the impact of temperature, which led to expansion of the Basquin's model

$$
\log \sigma_{a}\left(N_{f}, T\right)=\log a^{*}+b \log N_{f}+c \log T
$$

where: $a^{*}, b$ - parameters of the equation (constants of the model of regression), wherein $T-$ temperature at which tests are performed, $c$ - temperature-sensitive parameter $\mathrm{t}$

$$
c=\left.\frac{\partial \log \sigma_{a}\left(N_{f}, T\right)}{\partial \log T}\right|_{N_{f}=\text { const }}
$$

However, by assuming that the Basquin model is true for a certain reference temperature $T_{0}$, then equation (2.12) has a constant derived from the Basquin's model, and it can be written as

$$
\sigma_{a}\left(N_{f}, T\right)=a\left(\frac{T}{T_{0}}\right)^{c} N_{f}^{b}
$$

which means that $\sigma_{a}=a^{\prime} N_{f}^{b}$.

And this after logarithmisation gives as a result the Basquin's formula (Basquin, 1910).

The coefficients that are present in all models are determined by fitting the curve to experimental points. For this purpose the least square method is employed, and for non-linear models the Gauss-Newton algorithm. All characteristics can be drawn up on the basis of a single series of tests specified in the standards (Kurek et al., 2014).

Another characteristic that is shared by the mentioned in the introduction Section models and by the model developed by the authors is the fact that these models are based on stresses that occur in the material during fatigue tests. These values (of stresses), however, are empirical values, and thus they are not based on physical phenomena. While, for low cycle fatigue tests strain-based fatigue models considering both the plastic and elastic strains are applied; one of the most commonly used models is the Manson-Coffin-Basquin (MCB) fatigue life model

$$
\varepsilon_{a, t}=\varepsilon_{a, e}+\varepsilon_{a, p}=\frac{\sigma_{f}^{\prime}}{E}\left(2 N_{f}\right)^{b}+\varepsilon_{f}^{\prime}\left(2 N_{f}\right)^{c}
$$

where: $\varepsilon_{a, t}$ - strain amplitude expressed by the elastic strain amplitude $\varepsilon_{a, e}$ and plastic strain amplitude $\varepsilon_{a, p}, 2 N_{f}$ - reversal number, $E$ - Young's elastic modulus, $\sigma^{\prime} f, b$ - coefficient and exponent of the fatigue strength, respectively, $\varepsilon_{f}^{\prime}, c$ - coefficient and exponent of the plastic strain, respectively.

However, even in the case of strain-based fatigue characteristics, the problem of deficient mapping of real conditions can have a significant impact on the accuracy of the obtained characteristics. Such problem was described in (Marcisz, et al., 2012), the authors pointed on a certain strain value, which was not adopted in the model and which was for some materials so high that it could lead to serious errors while determining the characteristics. Type of this strain is neither plastic nor elastic. 



Fig. 1. Fatigue characteristics based on the proposed model designated for (a) St 42, (b) St 52-3, (c) $\mathrm{Ck} 45$ non-alloy steel

\section{Proposal of fatigue characteristics developed by the authors}

The proposed in the study stress-based fatigue characteristics model is designed to describe low and high cycle fatigue tests

$$
\log \frac{\sigma_{a}}{R_{m}}=B \log \left(2 N_{f}\right)+C \log ^{2}\left(2 N_{f}\right)+D \log ^{3}\left(2 N_{f}\right)
$$

where: $R_{m}$ - ultimate tensile strength, $B, C, D$ - constants.

In the model described by formula (3.1), it is assumed that the shape of stress-based fatigue characteristics in the extremely low cycle fatigue (ELCF) is dependent on the ultimate tensile strength $R_{m}$. The proposed solution considers the often noticed in research practic the fact that fatigue characteristics of most materials do not adopt in the double logarithmic system the shape of a straight line but it is rather like an inclined letter $S . B, C$ and $D$ constants adopted in formula (3.1) are determined numerically by the method of least squares. 

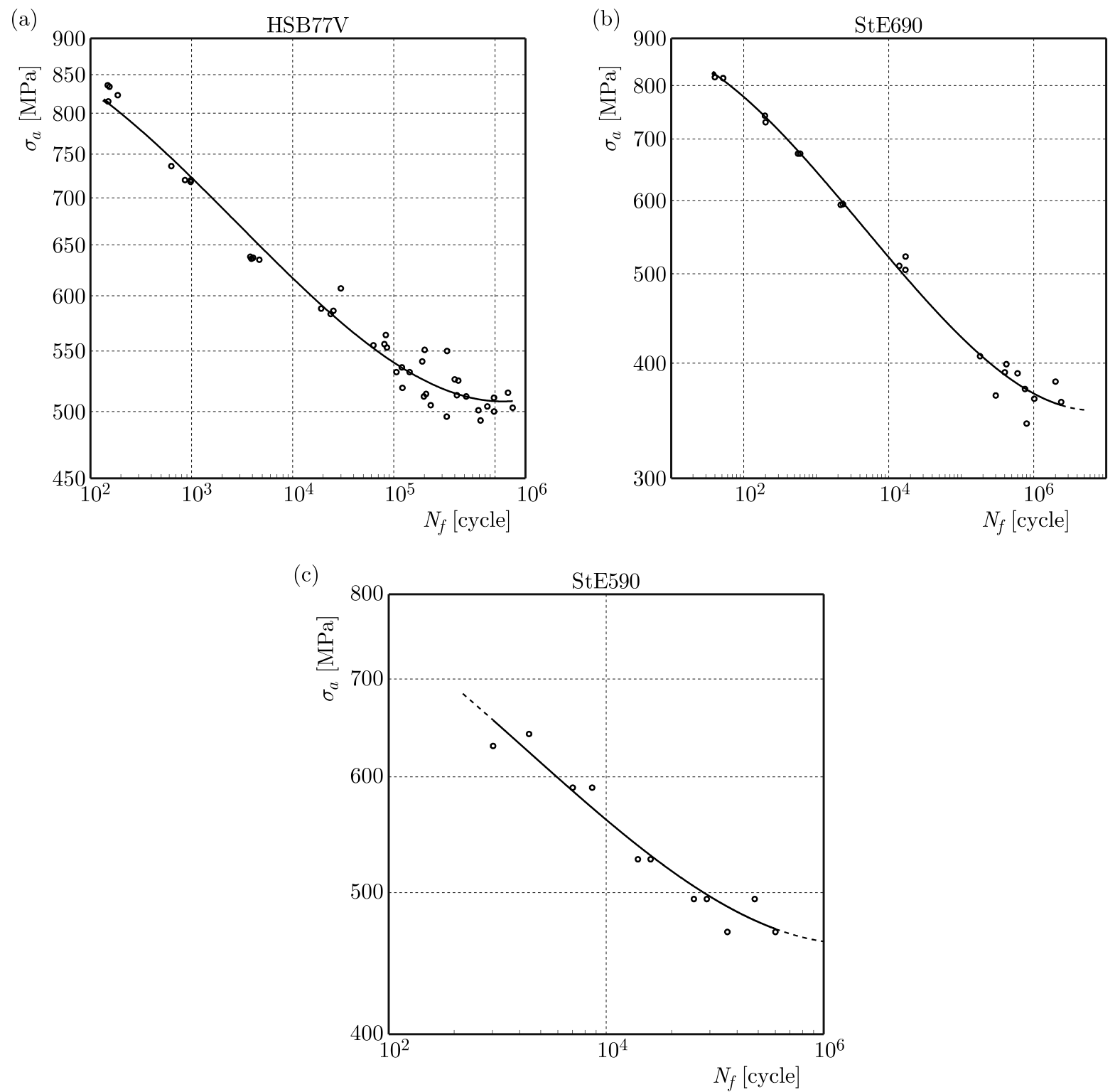

Fig. 2. Fatigue characteristics based on the proposed model designated for (a) HSB 77V, (b) St E690, (c) St E590 low-alloy steel

A similar approach to the one proposed by the authors on exponential characteristics was presented by Kandil (2000), Eq. (3.2). The model suggested by this author was indicated in (Kandil, 2000), however, it was applied to satisfy the objective of strain controlled fatigue research test. Therefore, these were strain-based fatigue characteristics

$$
\log \varepsilon_{a, t}=A-B \log N_{f}+C \log ^{2} N_{f}
$$

where: $\varepsilon_{a, t}-$ total strain amplitude.

\section{Verification of the proposed model}

Verification of the proposed model was performed on experimental results of ten various materials from three main types that were low-alloy, high-alloy and non-alloy steels. Fatigue test results were derived from research literature sources (Bäumel and Seeger, 1990). Table 1 lists materials 
(a)

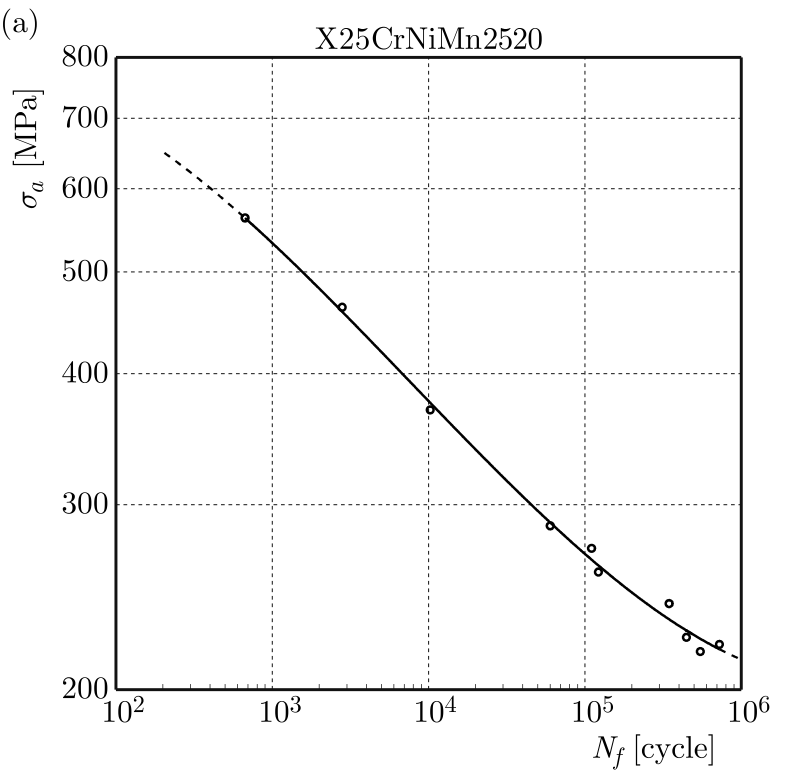

(a)

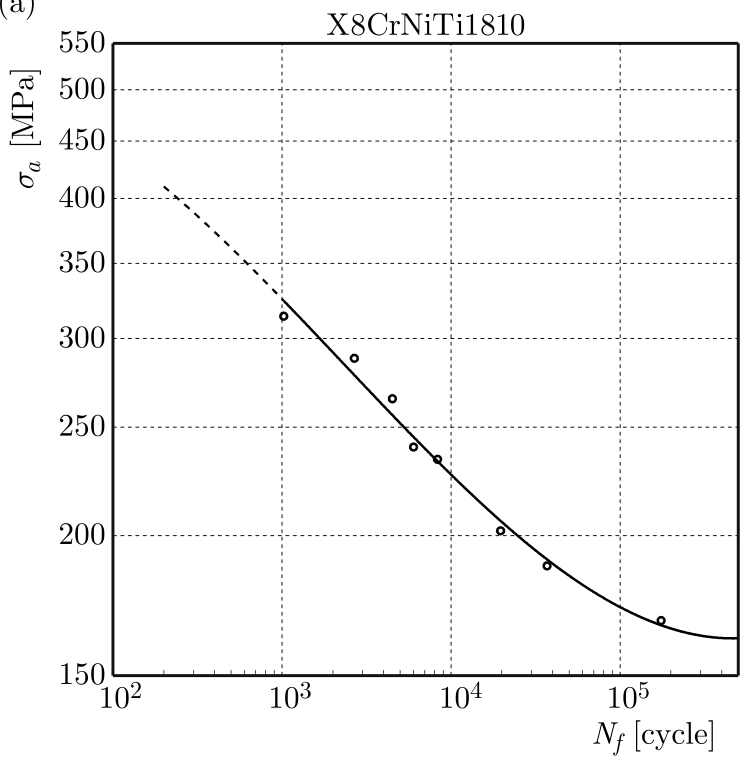

(b)



(b)

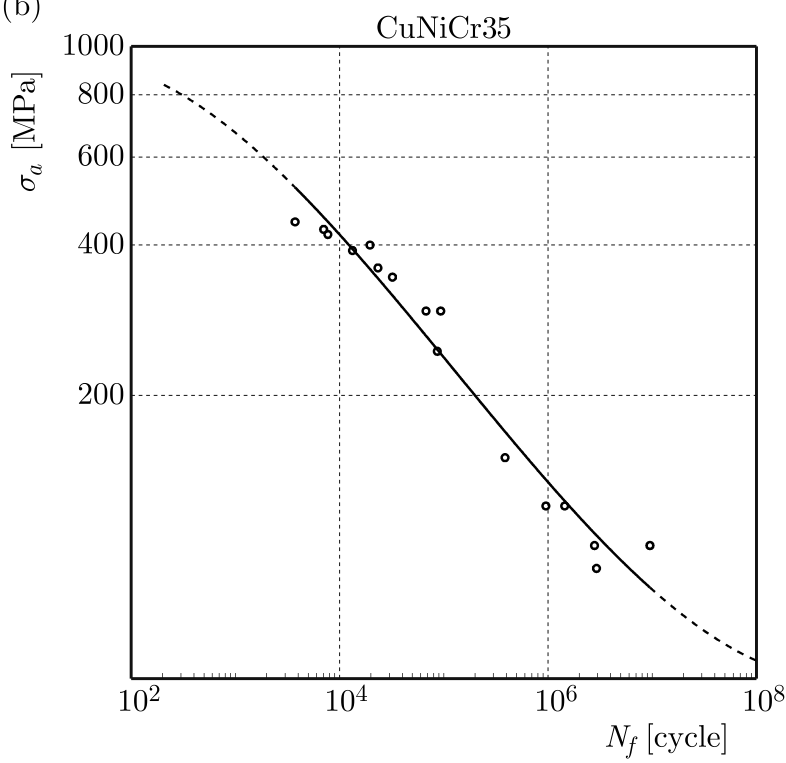

Fig. 3. Fatigue characteristics based on the proposed model designated for (a) X25CrNiMn2520, (b) X40CrMoV205, (c) X8CrNiTi1810, (d) CuNiCr35 high-alloy steel

used for verification of the model and basic properties of these materials, material constraints of the proposed model, Eq. (3.1), as well as the coefficient of determination $R^{2}$ estimated for characteristics of the material.

For all of the materials listed in Table $1, B, C$ and $D$ constants are designated as applied in the proposed formula (3.1). In this manner, the derived stress characteristics are presented as double logarithmic curves in Figs. 1-3. The continuous line indicates a section of characteristics as regards to experimentally designated points, whereas the dashed line presents an estimation of the characteristics performed based on Eq. (3.1) outside the scope of the experiment.

The performed simulation tests confirmed the possibility of application of the proposed model for describing fatigue properties of materials in the range from approx. 200 to 5000000 cycles as long as the model was used within the range of tested specimens. Applications of the model for specimens which broke during tests in lower number of cycles than 200 could cause some problems due to the fact that the ultimate tensile strength could be exceeded. 
Table 1. Summary table of tests performed on materials used for the analysis

\begin{tabular}{|c|c|c|c|c|c|c|c|}
\hline & Material type & $\begin{array}{l}\text { Young's } \\
\text { modulus } \\
E[\mathrm{GPa}]\end{array}$ & $\begin{array}{c}\text { Ultimate } \\
\text { tensile strength } \\
R_{m}[\mathrm{MPa}]\end{array}$ & $B$ & $C$ & $D$ & $R^{2}$ \\
\hline \multirow{3}{*}{ 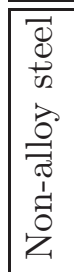 } & St 42 & 206 & 457 & 0.06016 & -0.03227 & 0.00244 & 0.9677 \\
\hline & St $52-3$ & 210 & 597 & 0.1157 & -0.05704 & 0.0048 & 0.9747 \\
\hline & CK 45 & 210 & 790 & 0.132 & -0.07743 & 0.00782 & 0.9447 \\
\hline \multirow{3}{*}{ 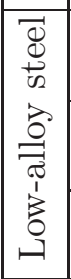 } & HSB 77V & 210 & 852 & 0.05023 & -0.03476 & 0.00336 & 0.9752 \\
\hline & St E690 & 214 & 872 & 0.03123 & -0.0343 & 0.00313 & 0.9889 \\
\hline & St E590 & 210 & 608 & 0.2805 & -0.157 & 0.01987 & 0.9462 \\
\hline \multirow{4}{*}{  } & X25CrNiMn2520 & 193 & 642 & 0.1455 & -0.07751 & 0.006665 & 0.9964 \\
\hline & X40CrMoV205 & 213 & 2576 & 0.1406 & -0.06579 & 0.005662 & 0.9772 \\
\hline & X8CrNiTi1810 & 181 & 411 & 0.1781 & -0.1006 & 0.009984 & 0.9894 \\
\hline & CuNiCr35 & 170 & 639 & 0.2422 & -0.09744 & 0.006372 & 0.9642 \\
\hline
\end{tabular}

\section{Conclusions and findings}

On the basis of the performed simulations for ten various materials, a statement can be formulated that the proposed model describes in a proper manner the results of experimental tests and it can be applied as fatigue characteristics for both the low and high cycle fatigue. Its basic advantage is the fact that it respects the tensile strength $R_{m}$ in order to reflect the nature of the stress curve, occurring under current conditions, which means its shape of an inclined letter $S$. We do not recommend using this model for the so called quasi-static range of fatigue life. The minimum number of cycles in simulations was appointed as 200. As in the most fatigue curve models, it is recommended to apply the authors' model only in the range of fatigue life corresponding with experimental data. Further analysis is required to fully prove the advantages and usefulness of the proposed model.

\section{Acknowledgement}

This work has been carried out under the grant of National Science Centre (Poland) No. 2015/19/B/ST8/01115.

\section{References}

1. Basquin O.H., 1910, The experimental law of endurance test, Proceedings of ASTM, 10, 625-630

2. Bastenaire F.A., 1972, New method for the statistical evaluation of constant stress amplitude fatigue-test results. Probabilistic aspects of fatigue, ASTM STP, $\mathbf{5 1 1}, 3-28$ 
3. Bäumel A., Seeger T., 1990, Material Data for Cyclic Loading, Supplement 1, Materials Science Monographs, 61, Elsevier Science Publishers, Amsterdam

4. Kandil F.A., 2000, The Determination of Uncertainties in Low Cycle Fatigue Testing, Standards Measurement \& Testing Project No. SMT4-CT97-2165, Issue 1, September, 1-26

5. Kato A., Hayashi M., 1999, Fatigue life estimation of steel using laser speckle sensor, NDT \& E International, 32, 139-145

6. Kohout J., 2000, Temperature dependence of stress-lifetime fatigue curves, Fatigue and Fracture of Engineering Materials and Structures, 23, 12, 969-977

7. Kurek M., Lagoda T., Katzy D., 2014, Comparison of fatigue characteristics of some selected materials, Materials Testing, 56, 2, 92-95

8. Marcisz E., NiesŁony A., Łagoda T., 2012, Concept of fatigue for determining characteristics of materials with strengthening, Fatigue Failure and Fracture Mechanics Book Series: Materials Science Forum, 726, 43-48

9. Palin-Luc T., 1996, Fatigue multiaxiale d'une fonte GS sous sollicitations combinées d'amplitude variable, These de doctorat, ENSAM Bordeaux

10. Pascual F.G., Meeker W.Q., 1999, Estimating fatigue curves with the random fatigue-limit model, Technometrics, 41, 277-302

11. Stromeyer C.E., 1914, The determination of fatigue limits under alternating stress, conditions, Proceedings of the Royal Society of London. Series A, Mathematical and Physical Sciences, 90, 411-425

12. WÖHLER A., 1858, Bericht über die versuche, welche auf der Königl. Niederschlesisch-Märkischen Eisenbahn mit Apparaten zum Messen der Biegung und Verdrehung von Eisenbahn-wagen-Achsen während der Fahrt, angestellt wurden, Zeitschrift für Bauwesen, 8, 642-652 